\section{Vergütung ohne Ende}

Ihren Glanzpunkt findet die gesundheitspolitische Debatte, wenn sie sich - von jedem störenden Inhalt befreit - nur noch mit dem Geld an sich beschäftigt. Werden bei der »Morbiditätsorientierten Gesamtvergütung « höchste Höhen erklommen, dreht sich in dieser Disziplin alles um Verfahren und Mathematik. Inzwischen kappt die Koalition mit den ambulanten Kodierrichtlinien für Ärzte die letzten Verbindungen zu realen Welt. Was bleibt ist morbide Geldschöpfung. Eine Chance, hier wieder an die reale Welt anzuknüpfen, in der Ärzte kranken Menschen helfen, können Verhandlungen vor Ort sein. Sie beziehen die unmittelbar Verantwortlichen ein, die auch reale Versorgungsprobleme lösen sollen. Dort findet »mehr Geld « wieder die Verbindung zu einer medizinischen Aufgabe, die besser gelöst werden soll. Die Stunde sich als Ärzteführer gerierender Honorarexperten ist vorbei: Ein FALK-land-Konflikt ganz eigener Art.

Sehr schmal ist der Grad, auf dem das bei den Wählern eifrig beworbene »Landarztgesetz « zur Vergütungsnovelle konvertiert. Nur der zwingende Abbau von Überversorgung in den Zentren kann mehr Ärzte aufs Land bringen: Woher sollen fehlende Ärzte sonst kommen? Aber die Koalition sieht die Stilllegung von Arztsitzen in überversorgten Bezirken und die zeitliche Befristung neuer Sitze nur als »Kann-Lösung « vor: Sie will dem nicht im Wege stehen. Kraftvolles Regieren geht anders. Im Endeffekt würde die neue Bedarfsplanung nur die bestehende Überversorgung verdecken und vereinzelte Unterversorgung unberührt lassen. Befeuert würde damit nur die honorarpolitische Argumentation. Und tatsächlich loben die Ärzteverbände euphorisch die beste Reform seit - mindestens 40 Jahren. Sie stört in keiner Weise, dass die notwendige Stärkung kooperativer Strukturen und partnerschaftlicher $\mathrm{Zu}$ sammenarbeit mit anderen Heilberufen erst gar nicht auf die Agenda kommt. Stattdessen unterfallen die MVZ der Restauration und die Aktiengesellschaft dem freiberuflichen Reinheitsgebot.

Besonders krass ist auch der Schwenk bei den spezialärztlichen Leistungen. Während die exorbitanten Mengensteigerungen beim Ambulanten Operieren - offenbar werden inzwischen vermehrt Gesunde operiert - ohne Antwort bleiben, versucht sich die Koalition an den hochspezialisierten Leistungen. Doch eine Lösung bettet der BMG weder in die neue Bedarfs- planung ein: Hier gerät der Landesausschuss in seiner sektorübergreifenden Form zu einer inhaltsleeren Hülse. Noch sucht er die Verbindung zu einem Vertragssystem. Der GKVSpitzenverband hatte Budgets vorgeschlagen, der AOK-Bundesverband einen Vorrang für Verträge mit der besten Qualitätssicherung. Der gefundene Weg zwischen den beiden Konzepten hindurch führt geradewegs in den von der DKG ausgerufenen »ordnungspolitischen Befreiungsschlag «: Also in die gähnende Leere - in ein regulatives Nichts. Auch die sektorübergreifende Qualitätssicherung des Bundesausschusses ist noch auf längere Sicht opperativ eine Luftbuchung. Und die titulierte » Bereinigung « der Gesamtvergütung hängt ohne vertragliche Grundlagen ebenso in der Luft wie die geforderte Wirtschaftlichkeitsprüfung, da sie auf keinerlei vertragliche Maßstäbe zurückgreifen kann. Zutreffend heißt es somit im Referentenentwurf »Die Krankenkasse zahlt unmittelbar «: Was wird ihr in dem Konstrukt auch anderes übrig bleiben. Gnädigerweise wird die Koalition ihre Jubelchöre wegen des langen Vorlaufes der Arbeiten im Bundesausschuss nicht selbst bezahlen müssen. So werden die Beteiligten die Sanierungswerkzeuge schon einmal bereit legen können für die Reform der Reform.

Das gilt in der Diktion der DKG zweifellos auch für den zweiten Befreiungsschlag. Bei der Reform des Bundesausschusses sollen Beschlüsse, die zum Ausschluss von Behandlungsmethoden aufgrund fehlenden Nutzens führen können, an eine Mehrheit von zwei Dritteln der Stimmen geknüpft werden. Für eine Republik, bei deren Geburt sich der schon betagte Kanzler mit der entscheidenden Stimme selbst gewählt hat, sind das durchaus gehobene Ansprüche. Von daher wird im Bundesausschuss künftig der langwierige Ausschluss nutzenfreier Methoden, gegen den sich die Deutsche Krankenhausgesellschaft bisher mit größter Zähigkeit zu widersetzen wusste, ohne großes Federlesen selbst ausgeschlossen sein. Die Beteiligten könnten sich zurücklehnen, da es damit auf der Beschlussebene kaum ein anderes Fazit als bisher gibt. Aber Vorsicht! Für die Großgeräte-, Medizinprodukte- und Diagnostikaindustrie wird die Markteintrittsschwelle auf diesem Wege ins Souterrain verlegt. Da nun für angebliche Innovationen nicht einmal mehr ein theoretisches Restrisiko besteht, dass Investitionen in nutzenfreie Methoden zumindest im Nachhinein abgeschrieben werden müssen, fehlt de facto nun auch die kaufmännische Selbstkontrolle der Firmen. Soviel Freiheit war nie. Der BMG hat in der Fachanhörung festgestellt, dass die Schwelle zum Strafrecht hier nicht überschritten werden soll. Die Anwendung nutzenfreier Methoden von der Körperverletzung zu unterscheiden ist eine Aufgabe besonderer Art. Immerhin gebietet die Aufklärung eine rationale Medizin, soweit sie nicht den Charme des Placebos beanspruchen kann. Und dafür wäre dieser ordnungspolitische Befreiungsschlag ja nun nicht erforderlich gewesen.

Die Herausgeber 